\title{
L'image de la science et des scientifiques sur les sites Internet de vulgarisation scientifique
}

The image of science and scientists on generalist science websites

\section{Laure Chémery et Laurent Billia}

\section{OpenEdition}

\section{Journals}

Édition électronique

URL : http://journals.openedition.org/ocim/111

DOI : 10.4000/ocim.111

ISSN : 2108-646X

Éditeur

OCIM

Édition imprimée

Date de publication : 1 septembre 2010

Pagination : 27-31

ISSN : 0994-1908

Référence électronique

Laure Chémery et Laurent Billia, «L'image de la science et des scientifiques sur les sites Internet de vulgarisation scientifique », La Lettre de l'OCIM [En ligne], 131 | 2010, mis en ligne le 01 septembre 2012, consulté le 01 mai 2019. URL : http://journals.openedition.org/ocim/111 ; DOI : 10.4000/ ocim. 111 


\section{L'image de la science et des scientifiques sur les sites Internet de vulgarisation scientifique}

\section{Laure Chémery et Laurent Billia *}

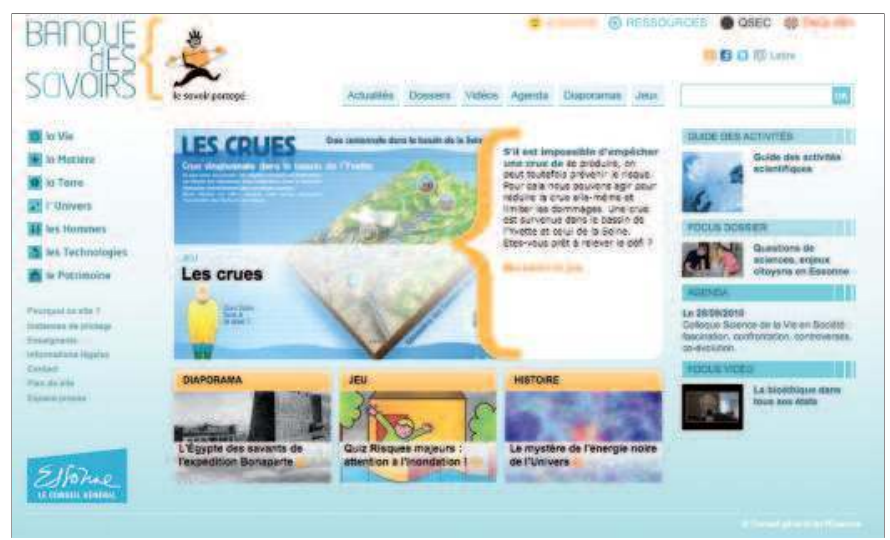

Un exemple français de site, La Banque des savoirs, dont les contenus scientifiques sont expertisés par des spécialistes.

* Laure Chémery est chef du service Accès aux savoirs au Conseil général de l'Essonne et coordinatrice du projet Yosciweb lchemery@cg91.fr

Laurent Billia est chef d'édition de la Banque des savoirs au Conseil général de l'Essonne et co-coordinateur du projet Yosciweb
Quelle image de la science et des scientifiques les sites de vulgarisation scientifique transmettent-ils aux jeunes? Voilà la grande question à laquelle six partenaires européens ont tenté de répondre sous la houlette du Conseil général de l'Essonne : deux ans après le début de cette investigation, intitulée Yosciweb (Young people and the images of sciences on websites) et financée par la Commission européenne, les résultats relèvent quelques paradoxes.

Depuis une dizaine d'années, un peu partout en Europe, on observe une décroissance notable de jeunes se dirigeant vers les filières scientifiques de l'enseignement supérieur. Ce constat ne manque pas d'inquiéter les pouvoirs publics, au premier rang desquels la Commission européenne, qui craignent de voir se tarir les impétrants aux carrières de chercheurs et d'ingénieurs. Cet état de fait est d'ailleurs corroboré par les études et enquêtes comme celles menées par Eurobaromètre qui montrent que, globalement, la perception de l'enseignement des sciences par les élèves est négative. Celui-ci est souvent considéré par les jeunes comme difficile et pas très pertinent pour la vie quotidienne, contrairement à la science en dehors de l'école considérée elle de manière plus positive.

C'est ainsi que début 2007, la DG Recherche lance, dans le cadre de son programme Sciences dans la Société (7e Programme cadre de recherche et développement) un appel à projet : comment améliorer l'image 
de la science et des scientifiques auprès des jeunes ? Comme le dit alors David Ros, vice président au Conseil général de l'Essonne chargé du Développement économique, de la Recherche et de l'Enseignement supérieur « la question de la désaffection des jeunes pour les sciences, manifeste au niveau des inscriptions universitaires, est un problème qui préoccupe les élus locaux : comment garantir le dynamisme économique d'un territoire si les jeunes se désintéressent de la science? Pour le Conseil général, ceci se traduit par l'édition de la Banque des savoirs d'une part et des liens renforcés avec les institutions en charge de l'Éducation nationale (rectorat et inspection académique) d'autre part. Autant dire que cet appel à projets représentait une vraie opportunité pour le Conseil général ».

Le Conseil général de l'Essonne, entouré de six autres institutions répond à l'appel de la manière suivante : si l'image de la science et des scientifiques auprès des jeunes est dégradée, dans le même temps, ceux-ci ont massivement adopté le média Internet. Voyons comment les sites Internet présentent la science et les scientifiques, comment les jeunes les perçoivent et, au delà de cette observation, voyons comment le média Internet modifie ou pas l'image de la science et des scientifiques. Deux ans après le début des travaux, quels sont les résultats les plus saillants?

\section{Trois observations sur l'image des scientifiques}

"Ce site montre que les scientifiques sont des personnes intelligentes» (Laetitia, 17 ans).

"Ce site montre que les scientifiques sont un peu dingos" (Nicolas, 16 ans) (1).

Première observation : où sont les chercheurs ? En effet, dans leur grande majorité, les sites Internet

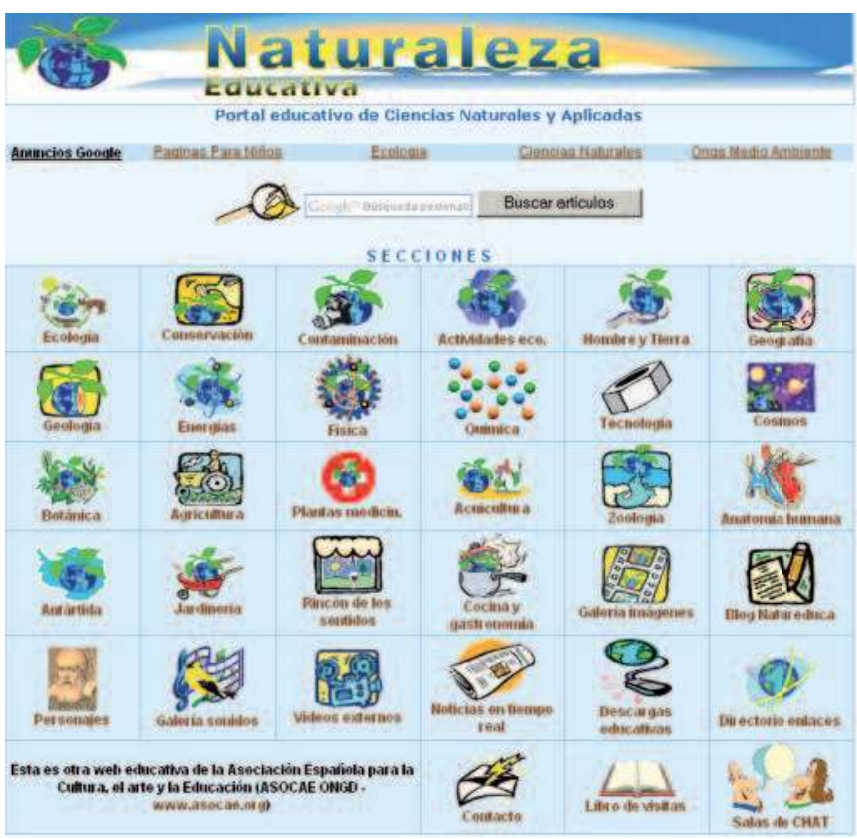

Le site espagnol dédié aux sciences naturelles et appliquées,

Natureduca, structure clairement sa page d'accueil sur des entrées thématiques correspondant aux sujets étudiés à l'école.

sélectionnés laissent peu de place aux scientifiques. Peu de place en images (photographies, films les montrant à l'œuvre...) mais aussi peu de place dans les contenus rédactionnels (nominations, interviews, citations, rédactions d'articles...) ou dans la gestion du site (comités scientifiques). La recherche scientifique, telle qu'elle est présentée par les sites Internet, est une activité le plus souvent désincarnée, mettant en œuvre de la pensée et de la technique, sans présence des corps. On peut alors s'interroger sur l'impact d'un tel parti pris (pas forcément délibéré) sur les jeunes qui ont besoin de concret, surtout lorsqu'il s'agit de se projeter dans un métier.

Deuxième observation : pourquoi les chercheurs présentés, quand ils le sont, sont-ils soit vieux et tristes,

\section{Le projet Yosciweb}

Le projet Yosciweb s'est déroulé de mars 2008 à mars 2010. Il a constitué en une succession d'étapes : repérage bibliographique, description du paysage Internet de chacun des pays concernés, sélection d'un échantillon de 60 sites (entre 6 et 10 par pays), mise au point de trois grilles d'analyse des sites (une grille pour l'image des scientifiques, une autre pour l'image de la science, une dernière pour les caractéristiques des site regroupées en 4 parties (contenu, graphisme, interactivité, ergonomie), analyse des 60 sites selon ces trois grilles, réalisation de
8 groupes de discussion par pays avec des jeunes de 11 à 18 ans, analyse des résultats des groupes de discussion, mise au point des tendances générales et initiatives singulières, élaboration des bonnes pratiques et recommandations.

L'ensemble des résultats, ainsi que les 6 lettres d'information présentant les résultats principaux, est disponible en anglais sur www.yosciweb.eu. Seuls les lettres d'information et le guide final ont été traduits dans les 6 autres langues du projet, dont le français. 
Yosciweb a été réalisé par 7 institutions situées en Bulgarie, Espagne, Estonie, France, Islande, PaysBas et Royaume-Uni.

Sur ces 7 institutions, 4 éditent un site de vulgarisation scientifique : Forum Democrit et Green Democrit (Democrit, Bulgarie), La Banque des savoirs (Conseil général de l'Essonne, France), Islandic web of science (Université de Reykjavik, Islande), Kennislink (Frije Universiteit von Amsterdam, Pays-Bas).

Les trois autres sont dédiées au thème « les jeunes et la science » : genre (Fundacio Centre d'Iniciatives
I Recerques, Catalogne, Espagne), pédagogie (Glasgow Caledonian University, Écosse, Royaume-Uni), innovation (Youth in Science and Business Foundation, Estonie).

Le consortium était assisté d'un comité scientifique indépendant chargé d'émettre des avis sur les protocoles comme sur les résultats. Il était composé de Yves Jeanneret, université d'Avignon, et de Carl Koppeschaar, expert indépendant à Haarlem, Pays-Bas. soit jeunes et jolies ? En effet, lorsque les sites laissent de la place aux scientifiques, il s'agit principalement d'hommes, âgés, en blouse blanche ou, plus rarement, de femmes jeunes, belles et dynamiques. Les sites Internet semblent osciller entre deux stéréotypes, le stéréotype classique, bien connu des jeunes et un contre stéréotype, semble-t-il destiné à contrecarrer le premier. Il est impossible de déterminer si ces représentations améliorent l'image de la science et des scientifiques ; en revanche, il est possible d'affirmer que ces stéréotypes masquent la diversité des métiers de la recherche et de ses acteurs.

Troisième observation : pourquoi les métiers de la science sont-ils merveilleux sur les sites de vulgarisation scientifique et « horribles » dans les médias généralistes ? Yosciweb s'est déroulé pour l'essentiel pendant la crise de la recherche. Le hiatus entre l'image véhiculée par les sites Internet de vulgarisation scientifique (métier passionnant...) et celle ressortant des médias d'information générale (questions des salaires, des statuts...) n'en était que plus criant. Or les jeunes sont parfaitement avertis des contraintes liées aux métiers de la recherche et répercutent très bien leurs interrogations concernant les salaires, la longueur des études, la précarité, les difficultés budgétaires. Là encore, il est difficile de déterminer l'effet de ce grand écart sur les jeunes. Mais la présentation d'informations pratiques sur les salaires, les emplois du temps, les congés... permettrait peut-être d'approcher un peu mieux la réalité.

\section{Trois observations sur l'image de la science}

"Ce site est chiant, trop scientifique. Il est utile pour le travail scolaire » (Théo, 15 ans)

"Ce n'est pas un sujet qui m'intéresse, et puis voilà!» (Laetitia, 17 ans)
Première observation : les sciences existent-t-elles ? Les jeunes ont une idée assez floue de ce que sont les sciences. Les plus jeunes ne voient pas du tout ce qu'elles sont, tandis que les plus âgés les réduisent aux matières scolaires. Mais on peut dire que les sites Internet dédiés aux sciences sont loin de clarifier la donne. Ils expliquent rarement quels sont leurs objectifs, la manière dont ils délimitent le champ thématique de la science/des sciences et leurs angles d'attaques. Par ailleurs, ils laissent peu de place à ce que pourrait être une science de proximité (pour les jeunes) hors espace et temps scolaire.

Deuxième observation : pourquoi les jeunes aimentils les sites de vulgarisation scientifiques mais détestent-ils les sciences ? Le présupposé de départ comme quoi la science aurait une image négative

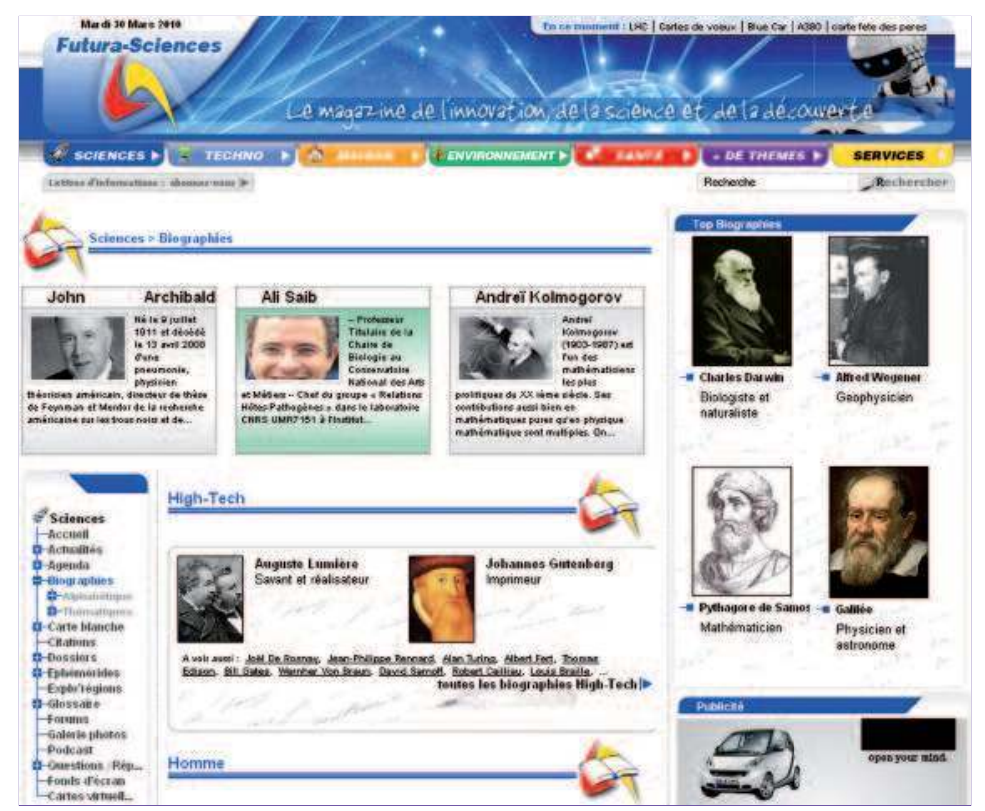

Futura-sciences, un exemple français de site qui mêle portraits de scientifiques contemporains et de personnages historiques. 
auprès des jeunes doit en fait être réduit aux matières scolaires. Or, ceci mène à une impasse car les jeunes ne consultent des sites Internet de vulgarisation scientifique que dans le cadre du travail scolaire. Pour les éditeurs de sites Internet, l'alternative n'est pas simple : doivent-ils répondre aux attentes en terme de scolarité, au risque d'être associés à l'école et donc jugés négativement ? Ou au contraire s'en éloigner, au risque de ne pas être consultés ?

Troisième observation : la science est-elle triste ? C'est en tout cas ce qui ressort de l'observation des sites Internet de vulgarisation scientifique qui sont le plus souvent caractérisés par l'austérité et la rigueur et qui s'autorisent rarement la fantaisie et l'humour. Notons à cet égard que les sites anglosaxons et néerlandais effectuent des choix éditoriaux plus audacieux et originaux, de fait difficilement imaginables sur les sites français.

\section{Trois observations sur les sites Internet de vulgarisation scientifique}

«Le site est facile à comprendre parce qu'il est animé » (Thomas, 12 ans)

"Je ne sais pas où se trouve l'information sur ce site" (Léa, 13 ans)

Première observation : les sites Internet existent-ils ? Du point de vue des jeunes, il s'agit d'une vraie question tant l'espace Internet est exploité comme un

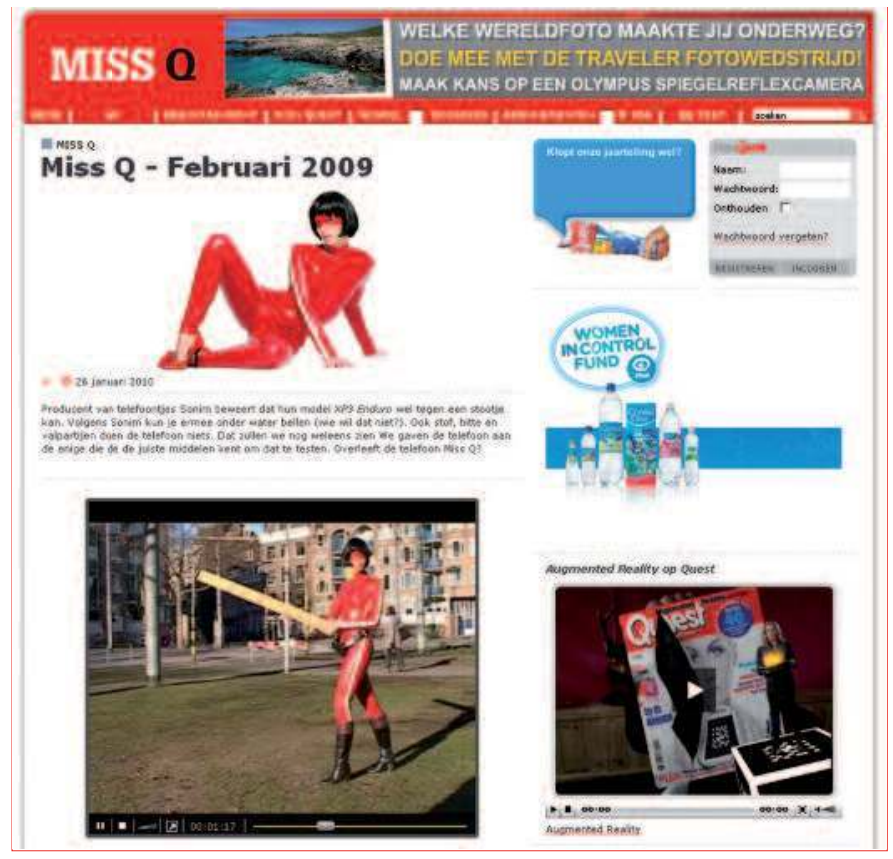

Un site néerlandais, Quest.nl, met en scène une sorte de James Bond Girl pour présenter des informations technologiques. vaste territoire de possibilités dans lequel ils naviguent au gré des moteurs de recherche et des liens rencontrés. Ils accèdent aux sites quasi-exclusivement par le biais des moteurs de recherche et en sortent parfois sans s'en rendre compte au gré des opportunités offertes par les liens. Par ailleurs, le jugement des jeunes est très influencé par le contexte dans lequel ils consultent le site : contexte scolaire ou contexte privé.

Deuxième observation : les sites Internet peuvent-ils prendre des libertés avec la science ? La science est un secteur dont les règles de fonctionnement sont explicites. Vérification des données, citations des sources, explicitation détaillée des protocoles suivis... pour ne citer que quelques-unes d'entre elles. D'une manière surprenante, les sites Internet de vulgarisation suivent rarement ces règles : les chartes éditoriales, les comités scientifiques et processus de validation semblent rares ou du moins sont rarement explicites. Si les jeunes ne sont pas a priori sensibles à ces questions (quoiqu'ils évoquent spontanément les questions de fiabilité des informations présentées sur Wikipédia), les importants prescripteurs que sont les enseignants apprécient, en revanche, que ces règles soient claires.

Troisième observation : pourquoi les jeunes plébiscitent-ils Wikipédia alors qu'ils disent n'aimer que les sites colorés, interactifs, animés ? Dans leur usage quotidien, les jeunes survalorisent les vidéos, les images, les animations... sans craindre d'affirmer que Wikipédia, pour le moins minimaliste en terme de graphisme, de multimédias et d'ergonomie, est leur site préféré en matière de sciences. Il est néanmoins certain que, dans tous les cas, les jeunes apprécient les sites structurés, organisés dans lesquels la navigation fonctionne intuitivement et sur lesquels il y a un moteur de recherche calqué sur le modèle Google.

\section{Convergences et divergences à l'échelle européenne}

Ce rapide extrait des observations, résultats et conclusions de Yosciweb ne doit pas masquer la complexité et la difficulté qu'il peut y avoir à les interpréter à l'échelle globale. Comme le rappelle Birgit Schroeter de l'université Calédonienne de Glasgow en charge de l'étude à l'échelle européenne des résultats des groupes de discussion : «les résultats sont très complexes. Par exemple, en Estonie et en Islande, les filles insistent sur l'utilité des sites tandis qu'en Espagne et en Bulgarie, ce sont les garçons qui 


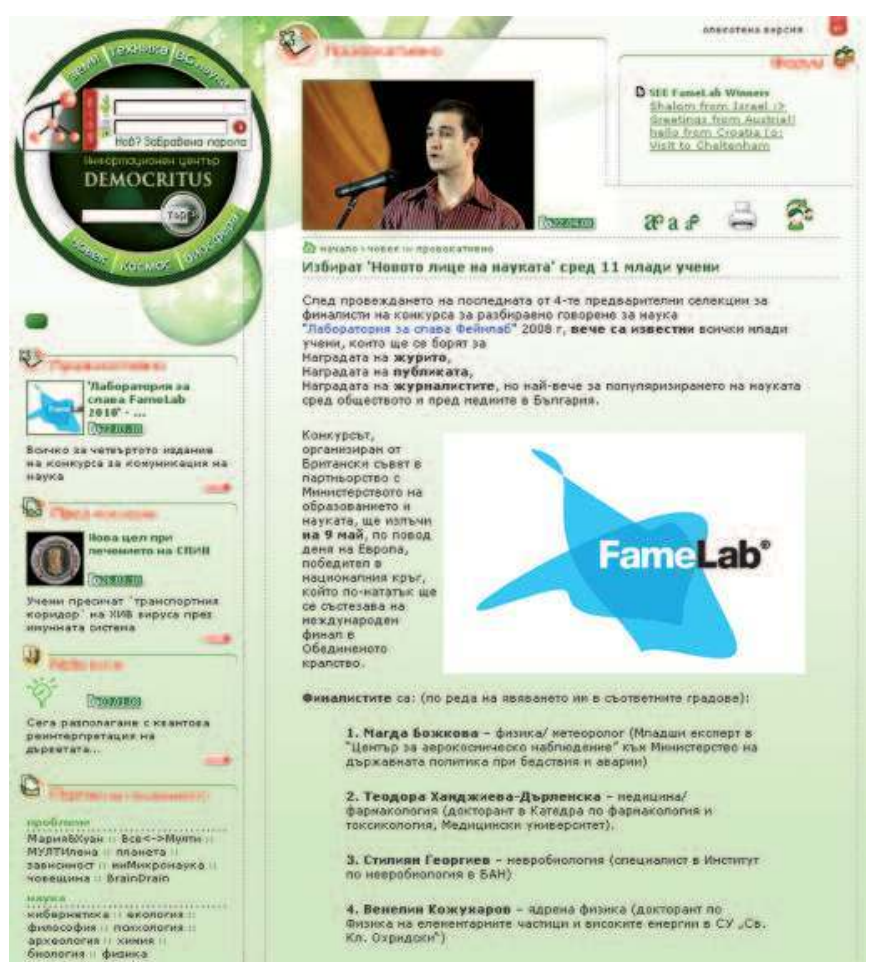

Un exemple bulgare, le site Green.democrit.com qui présente des portraits de jeunes chercheurs.

pointent ce critère. Cette différence peut être bien sûr liée à la variabilité des sites présentés mais aussi à des différences de comportement typiquement masculin ou féminin entre les groupes de pays ».

Voici quelques exemples de nuances nationales ou socio-culturelles qui peuvent être apportées aux résultats d'ensemble.

$\mathrm{Si}$ les poncifs concernant les scientifiques (un homme blanc, âgé, en blouse blanche, parfois un peu fou, travaillant beaucoup et gagnant peu d'argent) sont véhiculés par l'ensemble des jeunes rencontrés, en Bulgarie, les jeunes insistent sur l'honneur qu'il y a à être scientifique.

En Europe du Nord, les jeunes ont une vision plus méthodologique de la science qu'en France où elle est vue sous l'angle des disciplines (et particulièrement des matières scolaires).

À travers toute l'Europe, les jeunes les plus âgés et les jeunes issus de milieux socio-économiques plus favorisés, ont plus de facilités avec les sites, tant du point de vue navigation que du point de vue du contenu. Ceci peut être lié au taux d'équipement en ordinateurs à domicile.

Tous les sites Internet étudiés faisaient tous preuve d'un certain professionnalisme technique (ergonomie, charte graphique...). Néanmoins, les jeunes Estoniens et Bulgares se sont plaints, plus que ceux d'autres pays, des animations flash publicitaires et autres dispositifs imposés à l'internaute. Ceci est probablement lié au paysage local des sites Internet de vulgarisation scientifique et en particulier à la rareté de «sites publics».

Par ailleurs, d'un point de vue hexagonal, il semble que les sites néerlandais et britanniques s'autorisent plus de liberté (humour, graphisme, recours aux icônes populaires) que les sites français.

Deux ans de travail, 60 sites Internet étudiés sous toutes les coutures, quelques tendances lourdes et autant de spécificités nationales... Quelles seront les suites de ce projet? La promotion active des résultats a permis de toucher plusieurs centaines de personnes (journalistes, webmasters, enseignants, médiateurs scientifiques, représentants de centres de recherches...) au niveau européen. Chacun des partenaires directs du projet fera bien sûr son miel des résultats et observations du projet à l'échelle de son propre site. Enfin, le projet a permis de tisser des liens avec d'autres éditeurs de sites Internet, ce qui est peut-être l'amorce d'autres collaborations et échanges.

Les auteurs tiennent à remercier la Commission européenne, la DG Recherche, la Direction Sciences et société ainsi que les membres du consortium Yosciweb, à l'inspection académique de l'Essonne.

\section{Note}

(1) Toutes les citations sont extraites des groupes de discussion menés dans les collèges et lycées de l'Essonne. 